\title{
Bijections on m-level Rook Placements
}

\author{
Kenneth Barrese and Bruce E. Sagan \\ Department of Mathematics, Michigan State University, USA
}

\begin{abstract}
.
Partition the rows of a board into sets of $m$ rows called levels. An $m$-level rook placement is a subset of squares of the board with no two in the same column or the same level. We construct explicit bijections to prove three theorems about such placements. We start with two bijections between Ferrers boards having the same number of $m$-level rook placements. The first generalizes a map by Foata and Schützenberger and our proof applies to any Ferrers board. The second generalizes work of Loehr and Remmel. This construction only works for a special class of Ferrers boards but also yields a formula for calculating the rook numbers of these boards in terms of elementary symmetric functions. Finally we generalize another result of Loehr and Remmel giving a bijection between boards with the same hit numbers. The second and third bijections involve the Involution Principle of Garsia and Milne.
\end{abstract}

\section{Résumé.}

Nous considérons les rangs d'un échiquier partagés en ensembles de $m$ rangs appelés les niveaux. Un $m$-placement des tours est un sous-ensemble des carrés du plateau tel qu'il n'y a pas deux carrés dans la même colonne ou dans le même niveau. Nous construisons deux bijections explicites entre des plateaux de Ferrers ayant les mêmes nombres de $m$-placements. La première est une généralisation d'une fonction de Foata et Schützenberger et notre démonstration est pour n'importe quels plateaux de Ferrers. La deuxième généralise une bijection de Loehr et Remmel. Cette construction marche seulement pour des plateaux particuliers, mais ça donne une formule pour le nombre de $m$-placements en terme des fonctions symétriques élémentaires. Enfin, nous généralisons un autre résultat de Loehr et Remmel donnant une bijection entre deux plateaux ayant les mêmes nombres de coups. Les deux dernières bijections utilisent le Principe des Involutions de Garsia et Milne.

Keywords: Ferrers boards, Garsia-Milne Involution Principle, hit numbers, involutions, rook placements

\section{Introduction}

Rook theory is the study of the numbers $r_{k}(B)$, which count the number of ways to place $k$ non-attacking rooks on a board $B$. It originated with Kaplansky and Riordan who studied the connections between rook placements and elements of the symmetric group $S_{n}$ [KR46]. A Ferrers board is a board where the column heights form a weakly increasing sequence. Foata and Schützenberger characterized the equivalence classes of Ferrers boards by a unique, increasing, representative [FS70]. They did so by constructing explicit bijections between boards in a class and the unique representative. The rook polynomial of a board is the generating function for the $r_{k}(B)$ in the falling factorial basis for the ring of polynomials. The theorem of Foata and Schützenberger was later proved as an elegant corollary to the Factorization Theorem of Goldman, Joichi, and White [GJW75], which gave a complete factorization of the rook polynomial of a Ferrers board over the intergers. Loehr and Remmel constructed a bijection between rook placements on rook equivalent Ferrers boards using the Garsia-Milne Involution Principle which also implied the Factorization Theorem [LR09]. Later in the paper, they presented a similar bijection for the sets counted by the hit numbers of rook equivalent Ferrers boards. Briggs and Remmel generalized the notion of rook placements to $m$-level rook placements. These correspond to elements of $C_{m} 2 S_{n}$, the wreath product of the cyclic group of order $m$ with the symmetric group, in the same way that traditional rook placements correspond to elements of $S_{n}$ [BR06]. Using these placements and the concept of flag descents developed by Adin, Brenti, and Roichman [ABR01], Briggs and Remmel were able to generalize a formula of Frobenius to $C_{m} \imath S_{n}$.

The purpose of this abstract is to generalize the bijections of Foata and Schützenberger and those of Loehr and Remmel to $m$-level rook placements. The remainder of this section gives the background terminology necessary to begin this task. In Section 2 we generalize the bijections used by Foata and Schützenberger. Although these bijections arise as the composition of many intermediary bijections, and are therefore not direct, they do provide explicit bijections between $m$-level rook placements on arbitrary $m$-level rook equivalent Ferrers boards. We will 

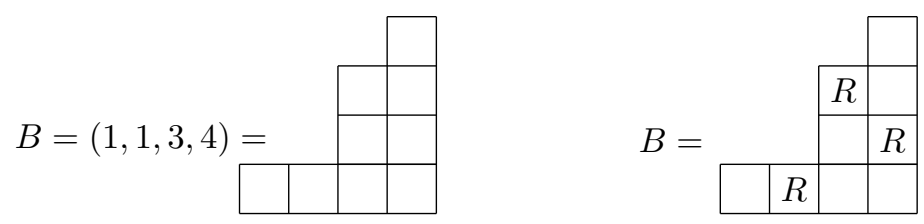

Fig. 1: A Ferrers board $B$ and a placement of three rooks on $B$.

need them again in Section 4 In Section 3 we generalize the construction of Loehr and Remmel. In this case the bijections can only be specified for singleton boards, a subset of all Ferrers boards. However, the construction leads to an explicit calculation of the $m$-level rook numbers for such boards using elementary symmetric functions and Stirling numbers of the second kind. In Section 4 , we generalize a second bijection of Loehr and Remmel, and in doing so prove that any two $m$-level rook equivalent Ferrers boards have the same hit numbers. The last two bijections involve the Garsia-Milne Involution Principle. Finally, in Section 5 we present an open problem of counting the number of Ferrers boards in $m$-level rook equivalence classes.

Given a partition $0 \leq b_{1} \leq b_{2} \leq \cdots \leq b_{n}$, a Ferrers board $B$ is the subset of $\mathbb{Z}^{+} \times \mathbb{Z}^{+}$given by

$$
B=\left\{(i, j) \in \mathbb{Z}^{+} \times \mathbb{Z}^{+} \mid j \leq b_{i}\right\} .
$$

Usually $B$ is denoted by $B=\left(b_{1}, b_{2}, \ldots, b_{n}\right)$. Graphically, one represents a Ferrers board as an array of square cells, where the $i$ th column contains $b_{i}$ cells. See the diagram on the left in Figure 1 for the board $(1,1,3,4)$. Throughout, we will use $c_{i, j}$ to denote the cell in the $i$ th column and $j$ th row of $B$. Note that this is neither the English nor the French style of writing Ferrers diagrams. However, it is useful because we usually place rooks rooks on the board from left to right and enumerating the number of such placements is facilitated by our convention.

For any non-negative integer $k$, a rook placement of $k$ rooks on $B$ is a subset of the cells of $B$ of cardinality $k$ which contains no more than one cell from any row or column of $B$. Graphically, this corresponds to placing rooks in the cells of $B$ so no two rooks attack each other. See the diagram on the right in Figure 1 for a placement of three rooks on $(1,1,3,4)$.

Henceforth we will assume that $m$ is a fixed positive integer. We define $\lceil j\rceil_{m}$ to be the least multiple of $m$ greater than or equal to $j$ and we call it the $m$-ceiling of $j$. Similarly, let $\lfloor j\rfloor_{m}$ be the greatest multiple of $m$ less than or equal to $j$, and call this the $m$-floor of $j$. Given a positive integer $p$, let $L_{p} \subset \mathbb{Z}^{+} \times \mathbb{Z}^{+}$be defined by:

$$
L_{p}=\left\{(i, j) \in \mathbb{Z}^{+} \times \mathbb{Z}^{+} \mid\lceil j\rceil_{m}=p m\right\} .
$$

Then the pth level of $B$ is $B \bigcap L_{p}$. Thus, the first level of $B$ consists of the first $m$ rows, the second level consists of the next $m$ rows, and so forth. Note that for $c_{i, j} \in B,\lceil j\rceil_{m}=p m$ if and only if $c_{i, j}$ is in the $p$ th level of $B$.

For any non-negative integer $k$, an m-level rook placement of $k$ rooks on $B$ is a subset of cardinality $k$ of the cells of $B$ which contains no more than one cell from any given level or column of $B$. See Figure 2 for two 2-level rook placements where thickened lines demarcate where levels begin and end; the numbering of the boards can be ignored for now. An $m$-level rook is a rook placed so that it is the only rook in its level and column. The $k$ th $m$-level rook number of $B$ is

$$
r_{k, m}(B)=\text { the number of } m \text {-level rook placements of } k \text { rooks on } B \text {. }
$$

Two boards are $m$-level rook equivalent if their $m$-level rook numbers are equal for all $k$. Note that $m$-level rook placements are always rook placements. Furthermore, when $m=1$ rook placements and $m$-level rook placements are equivalent.

The $i$ th column of $B$ terminates in level $p$ if $p$ is the largest integer such that the $i$ th column has non-empty intersection with $L_{p}$. A singleton board is any Ferrers board such that, for each positive integer $p$, the set of all columns terminating in level $p$ contains at most one column $i$ such that $b_{i} \not \equiv 0 \bmod m$. The Ferrers board on the left in Figure 2 is not a singleton board, as two different columns terminate in the second level without having 2 cells in that level, while the Ferrers boards in the middle and on the right are singleton boards.

\section{Rook equivalence and bijections}

In order to produce bijections between $m$-level rook placements on Ferrers boards, it is convenient to restrict our attention to singleton boards. In order to do this we prove the following two lemmas. First we show that for every 


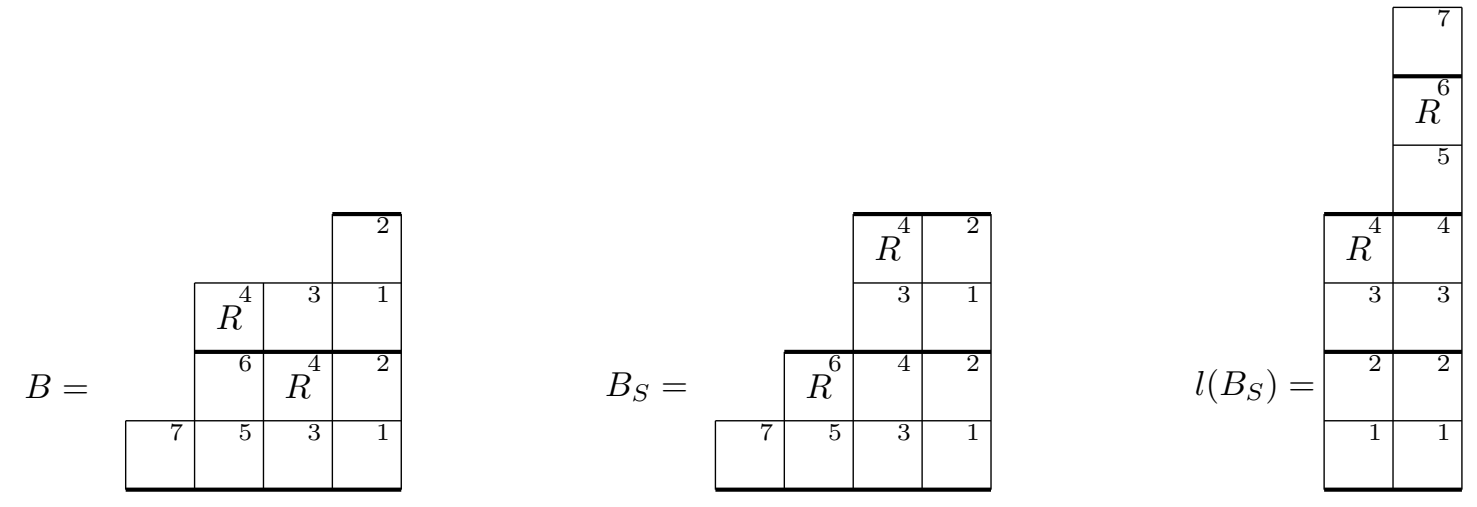

Fig. 2: On the left, a placement of two 2-level rooks on $B$. In the middle, the corresponding placement from Lemma 3 of two 2-level rooks on singleton board $B_{S}$. On the right, the placement on $l\left(B_{S}\right)$ from Lemma 5

Ferrers board there is a unique singleton board that has same number of cells at each level. Then we prove that there is a bijection between the rook placements on a Ferrers board and those on the singleton board guaranteed in the first lemma. These lemmas together imply that every Ferrers board is $m$-level rook equivalent to a singleton board and that there is an explicit bijection between the corresponding rook placements.

Lemma 1 Given a Ferrers board B, there exists a unique singleton board $B_{S}$ which has the same number of cells at each level as $B$.

Proof: (Sketch) The basic idea of the proof is to push all the cells in each level as far to the right as they can go keeping them in the level. This will create a singleton board because only the leftmost column in a level could contain fewer than $m$ cells, as any column to the right of that would recieve a full $m$ cells in order to put the cells as far to the right as possible. By construction, this singleton board will have the same number of cells at each level as $B$.

Ignoring the rook placement, Figure 2 shows a board $B$ and its corresponding board $B_{S}$. Since we know that an arbitrary Ferrers board $B$ has the same number of cells at each level as a unique singleton board $B_{S}$, we wish to provide an explicit bijection between rook placements on the two boards. In order to do so we require the following numbering on a Ferrers board.

Definition $2 A$ level numbering of board $B$ assigns a number to each cell of $B$ in the following way. Proceeding level by level in $B$, number the cells in the level by numbering each column from bottom to top, starting with the rightmost column and working left. In each level begin the numbering with 1.

Figure 2 presents two examples of this numbering, on the left and middle boards, and also illustrates the bijection of the next lemma.

Lemma 3 Given a Ferrers board B, there is an explicit bijection between m-level rook placements of $k$ rooks on $B$ and $m$-level rook placements of $k$ rooks on $B_{S}$, where $B_{S}$ is as constructed in Lemma 1 .

Proof: (Sketch) Start with the same construction as in Lemma 1. In addition, keep track of which cells originally contained rooks by using the level numbering, and place rooks in cells with the same number once they have moved. Because cells only get pushed to the right into columns that terminate at their level, if two rooks end up in the same column move the lower rook to the left into the column that the upper rook came from.

The combination of Lemma 1 and Lemma 3 guarantee that every Ferrers board is $m$-level rook equivalent to a singleton board. Additionally, there is an explicit bijection between $m$-level rook placements on the two boards. This permits us to restrict our attention to singleton boards henceforth.

Transposition of boards plays a central role in the Foata-Schützenberger construction of bijections between rookequivalent Ferrers boards when $m=1$. We will need a generalization of this operation for arbitrary $m$ and this is given in the next definition. 


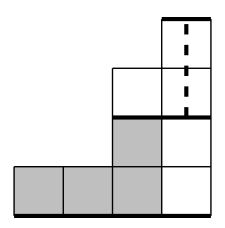

Fig. 3: The dashed line goes through the cells counted by the 2 -arm length of the fourth column and first level, and the shaded cells are counted by the corresponding 2-leg length.

Definition 4 Given a Ferrers board $B$ the l-operator applied to $B$ is defined as follows. If $t$ is largest index of a non-empty level of $B$ and the number of cells in the pth level of $B$ is $l_{p}$, then

$$
l(B)=\left(l_{t}, l_{t-1}, \ldots, l_{1}\right) .
$$

Figure 2 contains an example board $B_{S}$ as well as $l\left(B_{S}\right)$. The fact that $l(B)$ is a Ferrers board comes from the proof of Lemma 1, which shows that

$$
\left\lfloor l_{p}\right\rfloor_{m} \leq\left\lfloor l_{p-1}\right\rfloor_{m}
$$

with strict inequality if $l_{p} \not \equiv 0 \bmod m$. Thus, for any Ferrers board $B, l(B)$ will always be a Ferrers board and, in fact, a singleton board.

To see that the $l$-operator is a generalization of transposition, note that if $m=1$ then the levels of $B$ are individual rows and $l(B)$ is $B$ transposed. We now provide a bijection between rook placements on $B$ and rook placements on $l(B)$ to generalize the well known bijection for transposition.

Lemma 5 Given a singleton board $B$ and a non-negative integer $k$, there is an explicit bijection between $m$-level rook placements of $k$ rooks on $B$ and $m$-level rook placements of $k$ rooks on $l(B)$.

Proof: (Sketch) The basic idea is to take a rook from a level of $B$ to the corresponding column of $l(B)$. In order to determine how high up in the column of $l(B)$ the rook should go, give $B$ a level numbering and label each column of $l(B)$ from bottom to top. Move a rook so that the number of its cell is preserved. Since $B$ is a singleton board, the condition of having at most one rook in a column of $B$ is equivalent to having at most one rook in a level of $l(B)$.

Figure 2 illustrates this bijection. Note that Lemma 3 and Lemma 5 combine to provide an explicit bijection between $m$-level rook placements on any Ferrers board $B$ and on its $m$-transpose, $l(B)$. In practice it is simpler to consider only singleton boards, which we may do without loss of generality due to Lemma 3 .

For any set $S$, let \#S be the cardinatlity of $S$. Given a column $i$ and a level $p$ define the $m$-arm length of column $i$, level $p$ by

$$
\operatorname{arml}_{m}(i, p)=\#\left\{c_{i, j^{\prime}} \in B \mid c_{i, j^{\prime}} \text { is strictly above level } p\right\} .
$$

In Figure 3 the cells counted by the 2-arm length of column 4 , level 1 have a dashed line through them. (Reflecting our boards to put them in English notaiton will result in the arm being the usual set of squares when $m=1$.) We let $\operatorname{arml}_{m}(i, p)=\infty$ if the number of columns in $B$ is less than $i$ so that the equations in Definition 6 can still be used to test whether applying the $l$-operator locally in the last column of a board will result in a Ferrers board.

Similarly, define the $m$-leg length of column $i$, level $p$ to be

$$
\operatorname{legl}_{m}(i, p)=\#\left\{c_{i^{\prime}, j^{\prime}} \in B \mid c_{i^{\prime}, j^{\prime}} \text { is in level } p \text { and } i^{\prime}<i\right\} .
$$

The cells counted by the 2-leg length of column 4, level 1 are shaded in Figure 3 As before, this is equivalent to the usual notion of leg length in the $m=1$ case. We also let $\operatorname{legl}_{m}(i, 0)=\infty$ by convention. This is for similar reasons as the convention with $\operatorname{arml}_{m}$, this time for when the $l$ operator is applied locally to the first level of a board.

Since the $l$ operation generalizes the transposition of a Ferrers board, one would expect that some sort of local $l$ operation would be the appropriate generalization of the local transposition introduced by Foata and Schützenberger. This is indeed the case, and we define the local $l$ operation as follows.

Given a Ferrers board $B$ with non-empty intersection of the $i$ th column and $p$ th level, let $B_{i, p}$, denote the subboard of $B$ consisting of all cells in or above the $p$ th level and in or to the left of the $i$ th column, see Figures 4 and 5 for shaded examples. Note that if $B$ is a singleton board, then $B_{i, p}$ is also, because the set of rows in level $p^{\prime}$ of $B_{i, p}$ will be the same as the set of rows in level $p+p^{\prime}-1$ of $B$. If $B$ is a Ferrers board then the local $l$ operation at $(i, p)$ is 

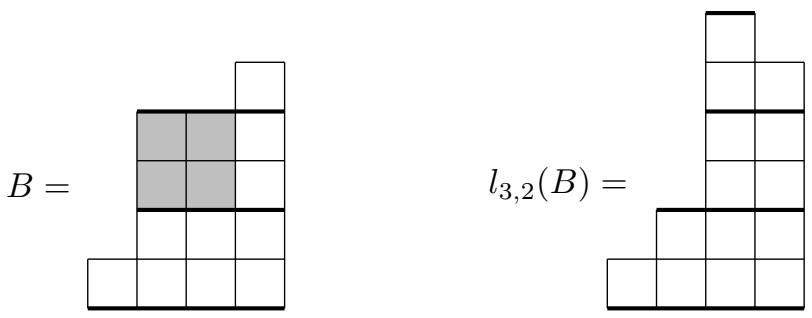

Fig. 4: On the left, $B_{3,2}$ is shaded within $B=(1,4,4,5)$. Notice that $l_{3,2}(B)$ is not permissible since $\left\lfloor\text { arml } l_{m}(4,2)\right\rfloor_{m}<$ $\operatorname{legl}_{m}(3,2)$ which means $l_{3,2}(B)$ will not yield a Ferrers board.

the result of applying the $l$ operator to the subboard $B_{i, p}$ and leaving the rest of $B$ fixed. We will denote the resulting board by $l_{i, p}(B)$.

As defined above $l_{i, p}(B)$ may not be a Ferrers board, let alone a singleton board. We now develop a pair of conditions to determine if $l_{i, p}(B)$ will be a singleton board.

Definition 6 The board $l_{i, p}(B)$ is permissible if

$$
\operatorname{arml}_{m}(i, p) \leq\left\lfloor\operatorname{legl}_{m}(i, p-1)\right\rfloor_{m} \quad \text { and } \quad \operatorname{legl}_{m}(i, p) \leq\left\lfloor\operatorname{arml}_{m}(i+1, p)\right\rfloor_{m} .
$$

See Figure 4 for an example of a board which is not permissible after applying a local $l$ operation, and Figure 5 for a local $l$ operation which produces a permissible board.

Lemma 7 Let singleton board B have a non-empty intersection of the ith column and pth level. If $l_{i, p}(B)$ is permissible then it is a singleton board.

Proof: (Sketch) The second permissibility condition implies that the columns of $l_{i, p}(B)$ are weakly increasing. The $m$-floor in the first condition together with the fact that $B$ is a singleton board ensures that the levels of $l_{i, p}(B)$ will each fit above the columns filled with a full $m$ cells in the level beneath.

Since there is a bijection between rook placements on $B$ and $l(B)$, it stands to reason that it would extend to a bijection between rook placements on $B$ and $l_{i, p}(B)$. The following lemma makes this precise.

Lemma 8 For a singleton board $B$, suppose $l_{i, p}(B)$ is permissible. Then there is an explicit bijection between $m$-level rook placements of $k$ rooks on $B$ and $m$-level rook placements of $k$ rooks on $l_{i, p}(B)$.

Proof: (Sketch) Begin with the bijection induced by the $l$ operation in Lemma 5 on the subboard transposed by $l_{i, p}$, not moving the rooks on the part of board $B$ which is fixed. There is a bijection between columns which gain a rook after applying the operator and columns which lose a rook. If two rooks end up in the same column, move the rook on the part of $B$ which is fixed to the corresponding column which lost a rook. The same is done with the levels.

Figure 5 illustrates this bijection. Foata and Schützenberger proved there is a unique Ferrers board in every rook equivalence class whose column lengths are strictly increasing and used this board as a target for their bijections. To accomplish the same thing, we need the following definition and theorem.

Definition 9 A Ferrers board $B=\left(b_{1}, b_{2}, \ldots, b_{n}\right)$ is called $m$-increasing if $b_{i+1} \geq b_{i}+m$ for all $1 \leq i \leq n-1$.

Notice that when $m=1$ increasing and $m$-increasing are equivalent.

Theorem 10 ([BLRS14] $)$ Every Ferrers board is m-level rook equivalent to a unique m-increasing board.

We are now almost ready to prove the main theorem. However, to do so we must put an order on Ferrers boards. Once we have established this order, we will be able to give an explicit bijection between an arbitrary Ferrers board $B$ and an $m$-level rook equivalent Ferrers board which is greater than $B$ in this order, if such a board exists. Additionally, the set of all Ferrers boards equivalent to $B$ will have a unique maximum element under this order, namely the $m$ increasing board guaranteed by [BLRS14].

To define this order, if $B=\left(b_{1}, \ldots, b_{n}\right)$ then consider the reversal of $B, B^{r}=\left(b_{n}, \ldots, b_{1}\right)$. Now let $B<B^{\prime}$ if $B^{r}$ is lexicographically smaller than $\left(B^{\prime}\right)^{r}$. It is important to note that when applying Lemma 3 we will always have

$$
B_{S} \geq B
$$

since in $B_{S}$ all the cells in each level are as far to the right as possible. 

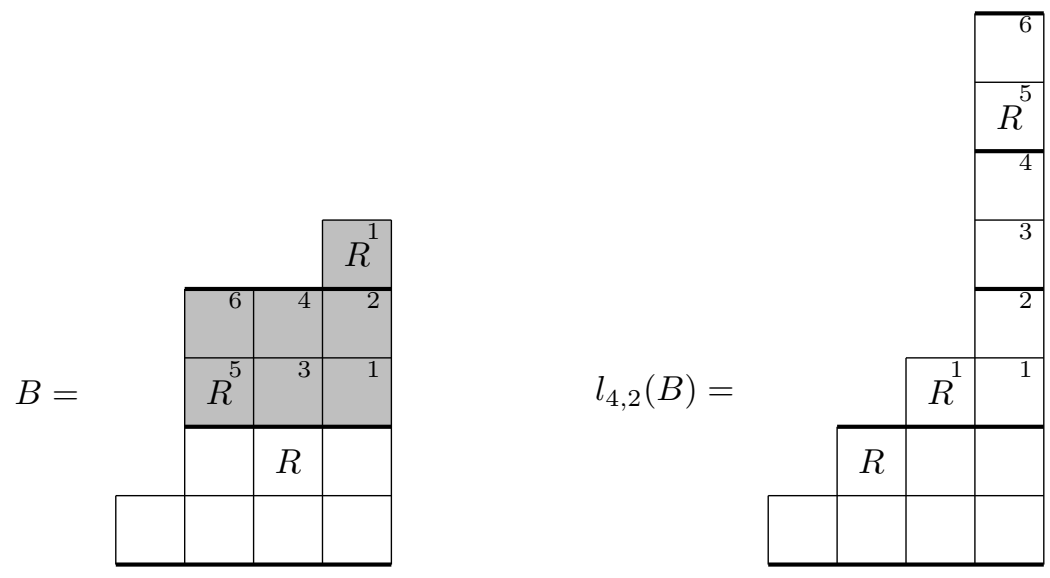

Fig. 5: On the left, $B_{4,2}$ is shaded. Here $l_{4,2}(B)$ is permissible and is shown on the right.

Lemma 11 Given a singleton board B containing a column $i$ and a level $p$ with the property that

$$
\operatorname{arml}_{m}(i, p)<\operatorname{legl}_{m}(i, p)
$$

there is a Ferrers board $B^{\prime}=l_{i^{\prime}, p}(B)$ with $i^{\prime} \geq i$ and $B^{\prime}>B$.

Furthermore, if $B$ is not m-increasing then a column $i$ and level $p$ satisfying equation 2.6 must exist.

Proof: (Sketch) If $i^{\prime}$ is the largest index of a column of $B$ satisfying the inequality 2.6, then one can use Lemma 7 to show that $B^{\prime}$ will be a Ferrers board. And this inequality also ensures that $B^{\prime}>B$.

For the second half of the lemma, if $B$ is not $m$-increasing then find a pair of columns $b_{i}, b_{i+1}$ with $b_{i+1}<b_{i}+m$. Then if $p$ is the top level of column $i+1$ which contains $m$ cells, $\operatorname{arml}_{m}(i+1, p)<\operatorname{legl}_{m}(i+1, p)$.

We are now in a position to prove our main theorem of this section.

Theorem 12 Given any two m-level rook equivalent Ferrers boards, there is an explicit bijection between m-level rook placements of $k$ rooks on them.

Proof: Given any Ferrers board $B$, let $B_{m}$ be the unique $m$-increasing board in the $m$-level rook equivalence class of $B$ guaranteed by Theorem 10. It suffices to show that there is an explicit bijection between the $m$-level rook placements of $k$ rooks on $B$ and those on $B_{m}$. This is trivial if $B=B_{m}$ so assume $B \neq B_{m}$. By Lemma 3, we have an explicit bijection between the placements on $B$ and those on $B_{S}$ where $B_{S} \geq B$ by equation 2.5. If $B_{S}=B_{m}$ then we are done. Otherwise, apply the local $l$ operator defined in Lemma 11 which will give $B^{\prime}=l_{i, p}\left(B_{S}\right)$ with $B^{\prime}>B_{S}$ and, by Lemma 8 , another explicit bijection between rook placements. We now repeat this process if necessary. Since there are only finitely many boards in an $m$-level rook equivalence class and the lexicographic order increases with every application of Lemma 8 , we must eventually terminate. And, by Lemma 11 again, termination must occur at $B_{m}$. Composing all the bijections finishes the proof.

See Figure 6 for a short example of this process.

(a)

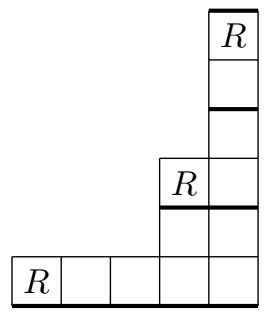

(b)

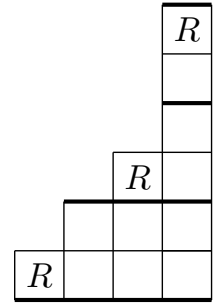

(c)

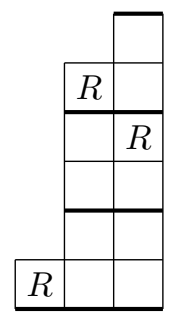

Fig. 6: (a) A 2-level rook placement on a Ferrers board. (b) The placement on the singleton board obtained after applying Lemma 3 (c) The placement obtained after applying Lemma 8 using $l_{3,1}$. Note that performing $l_{3,3}$ yields $B_{m}$. 

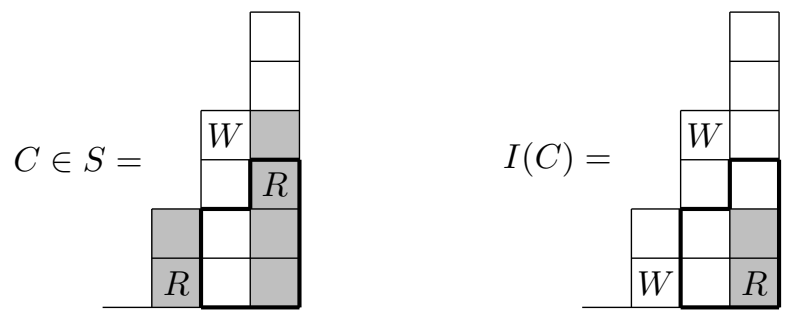

Fig. 7: On the left, an element in $S$ with sign -1 . On the right, the image under $I$ which has sign +1 .

\section{A second bijection on $m$-level rook placements}

Our next two main results will require the Garsia-Milne Involution Principle. The first will use the Involution Principle to construct another explicit bijection between two arbitrary $m$-level rook placements of $k$ rooks on $m$-level rook equivalent singleton boards.

Theorem 13 (Garsia-Milne [GM81]) Consider a triple $(S, T, I)$ where $S$ is a signed set, $I$ is a sign-reversing involution, and $T$, the set of fixed points of $I$, is required to be a subset of the positive part $S^{+}$of $S_{\text {. Let }}\left(S^{\prime}, T^{\prime}, I^{\prime}\right)$ be defined similarly. Then, given a sign-preserving bijection $f$ from $S$ to $S^{\prime}$, one can construct an explicit bijection between $T$ and $T^{\prime}$.

The way that Garsia and Milne define the explicit bijection is as follows. Start with an element $t \in T \subseteq S^{+}$. If $f(t) \notin T^{\prime}$, then apply $\left(f \circ I \circ f^{-1} \circ I^{\prime}\right)$ to $f(t)$. This takes $f(t) \in S^{\prime+}$ to $S^{\prime-}$, then to $S^{-}$, then $S^{+}$, and finally back to $S^{\prime+}$. Iterating this procedure must ultimately yield an element of $T^{\prime}$ which is considered the image of $t$ under the desired bijection.

We will use the Involution Principle to construct a bijection between $m$-level rook placements on two $m$-level rook equivalent singleton boards. We must first construct a signed set and a sign-reversing involution so that the $m$-level rook placements are the fixed points under the involution. We do this as follows.

Given two Ferrers boards, $B$ and $B^{\prime}$, we shall say $B$ fits inside $B^{\prime}$ if juxtaposing the two boards with their lower right cells in the same position makes the cells of $B$ a subset of the cells of $B^{\prime}$. Figure 7 shows that the thick bordered $B=(2,3)$ fits inside $B^{\prime}=(0,2,4,6)$. The shading and placement may be ignored for now. Let $\Delta_{n, m}$ denote the triangular Ferrers board $(0, m, 2 m, \ldots,(n-1) m)$. Given a singleton board $B$, fix $N$ large enough that $B$ fits inside $\Delta_{N, m}$. If $B$ has fewer than $N$ columns, expand $B$ on the left with columns of height zero so $B=\left(b_{0}, b_{1}, \ldots, b_{N-1}\right)$ has the same number of columns as $\Delta_{N, m}$. Fix a non-negative integer $k$ with $k<N$ and let the integer $i$ vary over $0 \leq i \leq k$. Then $S$ will consist of all configurations $C$ constructed as follows. Take $\Delta_{N, m}$ with $B$ fitting inside and place white rooks $W$ in $i$ cells of $\Delta_{N, m}$ that are outside of $B$ so that no two white rooks are in the same column. Next, place $k-i$ black rooks $R$ forming an $m$-level rook placement on the subboard $\Delta_{N-i, m}$ which is located in the columns of $\Delta_{N, m}$ which do not contain a white rook. We will call this the inset board. Note that the columns of the inset $\Delta_{N-i, m}$ may not be contiguous.

See the left board of Figure 7 for an example of such an object $C$ where $m=2$. The singleton board $B=(0,0,2,3)$ fits inside $\Delta_{4,2}$. Here $k=3<4$ and there is $i=1$ white rook on the board $\Delta_{4,2} \backslash B$ and $(k-i)=2$ black rooks on the board $\Delta_{3,2}$ which is represented by the grey shaded cells inside $\Delta_{4,2}$. The rooks on $\Delta_{3,2}$ form a 2-level rook placement, but there is both a black rook and a white rook in the second level of $\Delta_{4,2}$.

Note that each column of $\Delta_{N, m}$ may contain at most one white rook or black rook. On the other hand, a level of $\Delta_{N, m}$ will contain at most one black rook, but may contain any number of white rooks. Further, define the sign of such a placement to be $(-1)^{i}$. The sign of the placement on the left in Figure 7 is -1 .

To define $I$ on an element $C \in S$, if all rooks of $C$ are in $B$, and therefore black, then $C$ is a fixed point. Otherwise, examine the columns of $C$ from left to right until coming to a column with a rook outside of $B$. If that column contains a black rook, change the rook to a white rook, increase $i$ by one, and move every black rook above and to the right of the cell containing the new white rook down $m$ cells. If the column contains a white rook, change it to a black rook, decrease $i$ by one, and move every black rook to the right and the same level or higher as the new rook up $m$ cells. The placement on the right in Figure 7illustrates what happens to the board on the left under $I$. Similarly, $I$ takes the placement on the right to the placement on the left. 

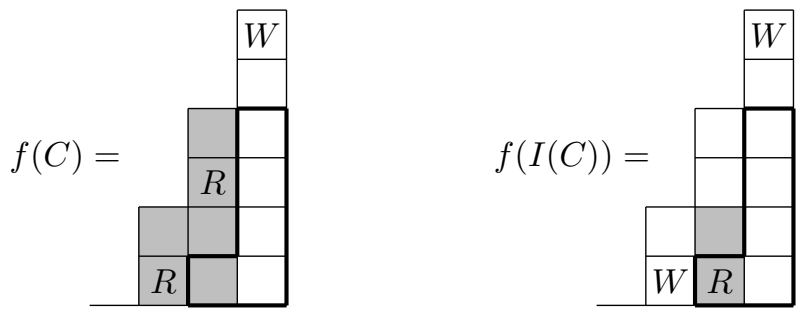

Fig. 8: The image under $f$ of the boards from Figure 7 on the left and the right respectively.

We must show that $I(C)$ will be an element of $S$. Clearly each column has at most one rook. Each level will have at most one black rook since when a black rook is added all black rooks at its level or above to the right of the new rook move up one level. Furthermore, if we change a white rook to a black rook, there can be no black rooks at the same level or higher to the left of the new black rook. This is because the new black rook was a white rook which, by definition, was above board $B$. Since $B$ is a singleton board, no columns to the left of the white rook in question will terminate in the level of the white rook. Thus if there were a black rook at the same level or higher to the left, it too would be outside of board $B$, which contradicts the white rook being the leftmost rook outside of board $B$.

When a black rook is added, the black rooks must be placed on a board $\Delta_{N-i+1, m}$ where the column in which the new black rook is placed is added to the columns in the initial inset $\Delta_{N-i, m}$. Since there are no white rooks to the left of the new black rook, there will be no omitted columns to the left of the column containing the new black rook, thus all cells of that column will be in the inset $\Delta_{N-i+1, m}$ and the new rook must be inside $\Delta_{N-i+1, m}$. This means that all the columns to the right of the new black rook that do not contain a white rook will contain $m$ more squares of the inset $\Delta_{N-i+1, m}$ than they did of the $\Delta_{N-i, m}$. Thus moving black rooks to the right of the new black rook up $m$ cells will keep them within the new $\Delta_{N-i+1, m}$. Similarly, changing a black rook to a white rook will decrease the number of cells in the columns of $\Delta_{N-i-1, m}$ to the right of the new white rook by $m$, but all black rooks to the right of the new white rook and at a higher level than it are moved down $m$ cells, so they will be in $\Delta_{N-i-1, m}$ because they were in $\Delta_{N-i, m}$ originally. Finally, if there are any black rooks below the level of the new white rook but to its right, they will remain in $\Delta_{N-i-1, m}$ because the first column in $\Delta_{N-i-1, m}$ to the right of the new white rook must go up to at least the level of the new white rook, because previously it was a black rook contained in $\Delta_{N-i, m}$.

By construction, $I$ is an involution. The fixed set of $I$ will be denoted $T$. It is the set of all configurations which only have rooks on the subboard $B$ and, by definition, these rooks must be black. As such, $T$ is equal to the set of $m$-level rook placements of $k$ rooks on $B$. Furthermore, if a board is not in $T$, then $I$ either increases or decreases the number of white rooks on the board by one. Either way $I$ will change the sign of the board.

Given a singleton board $B^{\prime}$, define $N^{\prime}, S^{\prime}, t^{\prime}$, and $I^{\prime}$ similarly for $B^{\prime}$ contained in $\Delta_{N^{\prime}, m}$. Without a loss of generality, assume $N=N^{\prime}$. Let $B^{\prime}=\left(b_{0}^{\prime}, b_{1}^{\prime}, \ldots, b_{N-1}^{\prime}\right)$. If $B$ and $B^{\prime}$ are $m$-level rook equivalent singleton boards we can use $I$ to construct an explicit bijection between $m$-level rook placements of $k$ rooks on $B$ and $m$-level rook placements of $k$ rooks on $B^{\prime}$. We do this by constructing a sign-preserving bijection between $S$ and $S^{\prime}$. We will need the following characterization of when two singleton boards are $m$-level rook equivalent.

The root vector of $B$ is

$$
\zeta_{m}=\left(b_{0}, m-b_{1}, \ldots,(N-1) m-b_{N-1}\right) .
$$

The following result of Briggs and Remmel determines when two singleton boards are $m$-level rook equivalent simply by considering their root vectors.

Theorem 14 (Briggs-Remmel [BR06]) Two singleton boards are $m$-level rook equivalent if and only if they have the same root vector up to rearrangement for a sufficiently large $N$.

We are now ready to apply the Garsia-Milne Involution Principle.

Theorem 15 Let $B$ and $B^{\prime}$ be m-level rook equivalent singleton boards. Then there exists an explicit bijection between $m$-level rook placements of $k$ rooks on $B$ and m-level rook placements of $k$ rooks on $B^{\prime}$. 


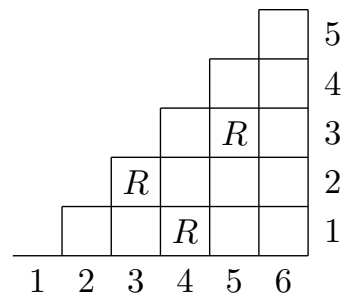

Fig. 9: The placement corresponding to partition $\{1,4\},\{2,3,5\},\{6\}$.

Proof: By Theorem 13, it suffices to find a sign-preserving bijection $f: S \rightarrow S^{\prime}$ since our involution has as $T$ the set of $m$-level rook placements of $k$ rooks on $B$ and as $T^{\prime}$ the set of $m$-level rook placements of $k$ rooks on $B^{\prime}$. We construct $f$ as follows.

For clarity of notation, let $B$ be placed in $\Delta_{N, m}$ and $B^{\prime}$ be placed in a copy $\Delta_{N, m}^{\prime}$ of $\Delta_{N, m}$. Notice that the $k$ th element of the root vector of $B, k m-b_{k}$, is the number of cells in the $k$ th column of $\Delta_{N, m}$ which lie outside of board $B$. Since $B$ and $B^{\prime}$ are $m$-level rook equivalent the root vector for $B^{\prime}$ is a rearrangement of the root vector for $B$. Therefore there is a canonical, length-preserving bijection between the columns of the set difference $\Delta_{N, m} \backslash B$ and the columns of $\Delta_{N, m}^{\prime} \backslash B^{\prime}$. This bijection induces a bijection on the placement of the white rooks. If a white rook appears in the $j$ th cell above $B$, place a white rook in the $j$ th cell above $B^{\prime}$ in the associated column.

Once all the white rooks are placed, create a copy of $\Delta_{N-i, m}^{\prime}$ inside of $\Delta_{N, m}^{\prime}$ using the columns which do not contain a white rook. Place the black rooks on the board with relation to the $\Delta_{N-i, m}^{\prime}$ subboard exactly as they are placed on the original board with relation to the original $\Delta_{N-i, m}$ subboard. Figure 8 illustrates the placements for $B^{\prime}=(0,0,1,4)$ corresponding to the two placements from Figure 7 for $B=(0,0,2,3)$. Notice that in the left board in Figure 7, the white rook is at the top of the second column from the left which has two cells above $B$. In Figure 8 the white rook is still at the top of the second column from the left which has two cells above $B^{\prime}$.

Under this map the white rooks must be placed inside $\Delta_{N, m}^{\prime}$ but outside $B^{\prime}$, the black rooks are placed inside $\Delta_{N-i, m}^{\prime}$, so it maps $S$ to $S^{\prime}$. Further this map preserves the number of white rooks placed on the board, so it is clearly sign preserving. Therefore we may conclude from the Involution Principle that there is an explicit bijection between $m$-level rook placements of $k$ rooks on $B$ and placements $m$-level rook placements of $k$ rooks on $B^{\prime}$.

To obtain a consequence of this construction, we will need some background on symmetric functions and Stirling numbers. For $d \leq n$ both non-negative integers, let $e_{d}\left(x_{1}, x_{2}, \ldots, x_{n}\right)$ denote the elementary symmetric function of degree $d$ in $n$ variables, that is,

$$
e_{d}\left(x_{1}, x_{2}, \ldots, x_{n}\right)=\prod_{1 \leq i_{1}<i_{2}<\cdots<i_{d} \leq n} x_{i_{1}} x_{i_{2}} \ldots x_{i_{d}} .
$$

Let $S(n, d)$ denote the Stirling numbers of the second kind. Recall that $S(n, d)$ can be defined as the number of ways to partition a set of $n$ elements into $d$ subsets called blocks.

Further, note that $S(n, d)$ counts the number of rook placements of $n-d$ rooks on $\Delta_{n, 1}$. To see this, number the rows of $\Delta_{n, 1}$ from 1 to $n-1$ from bottom to top. Then number the columns, including the zero column, from 1 to $n$ left to right. Given a partition of $\{1, \ldots, n\}$ into $d$ blocks, order the elements of each block increasingly. Now, if $i$ and $j$ are adjacent within a block then place a rook in row $i$ column $j$. See Figure 9 for the rook placement corresponding to $\{1,4\},\{2,3,5\},\{6\}$. Thus the number of $m$-level rook placements of $(n-d)$ rooks on $\Delta_{n, m}$ is $m^{n-d} S(n, d)$. The extra $m^{n-d}$ counts the number of ways of choosing a placement for each of the $(n-d)$ rooks in the $m$ cells of a level.

It is interesting to note that the construction of $I$ yields the following theorem giving an explicit calculation for the $m$-level rook numbers of a singleton Ferrers board $B$.

Theorem 16 For any singleton board $B=\left(b_{0}, b_{1}, \ldots, b_{N-1}\right)$,

$$
r_{k, m}(B)=\sum_{i=0}^{k}(-1)^{i} m^{k-i} S(N-i, N-k) e_{i}\left(-b_{0}, m-b_{1}, \ldots,(N-1) m-b_{N-1}\right) .
$$

Proof: Since the fixed points of the involution $I$ are counted by $r_{k, m}(B)$, it suffices to show that the sum counts all elements of the set $S$ by sign. First note that the number of ways of putting $i$ white rooks in $i$ unique columns of $\Delta_{N, m}$ 


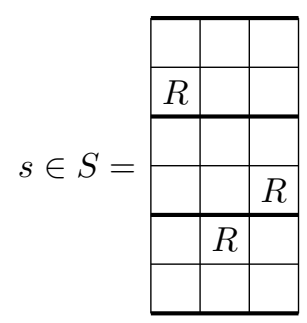

Fig. 10: The placement on $\mathrm{Sq}_{3,2}$ corresponding to $\left(\alpha^{1}, \alpha^{2}, \alpha^{1} ;(1,3,2)\right)$.

outside of $B$ is $e_{i}\left(-b_{0}, m-b_{1}, \ldots,(N-1) m-b_{N-1}\right)$. Furthermore the number of $m$-level rook placements of $k-i$ rooks on $\Delta_{N-i, m}$ is $m^{k-i} S(N-i, N-k)$. Putting these two counts together with the appropriate sign gives the sum as desired.

Note that this theorem implies the backward direction of Theorem 14

\section{A bijection for hit numbers}

We will now use the Involution Principle to prove that two boards that are $m$ level rook equivalent have the same hit numbers. We begin with some definitions. As usual, let $m$ be a fixed positive integer. We will sometimes suppress $m$ in the following notation to limit the number of subscripts.

Let $B$ be a Ferrers board and let the integer $N$ be sufficiently large so that $B$ fits inside a rectangular board, $\mathrm{Sq}_{N, m}$ with $N$ columns and $m N$ rows. If $\alpha$ is a generator of the cyclic group $C_{m}$, then

$$
C_{m} \imath S_{N}=\left\{\left(\alpha^{s_{1}}, \alpha^{s_{2}}, \ldots, \alpha^{s_{N}} ; \sigma\right) \mid 1 \leq s_{i} \leq m \text { for each } i \text { and } \sigma \in S_{N}\right\} .
$$

We associate with $\omega \in C_{m} \curlywedge S_{N}$ a placement on $\mathrm{Sq}_{N, m}$ by placing a rook in level $p$ and column $i$ if $\sigma(i)=p$. Furthermore, the rook in column $i$ will be $j$ cells from the bottom of the level if $s_{i}=j$. See Figure 10 for an example with $m=2$ and $N=3$, where the placement corresponds to $\left(\alpha^{1}, \alpha^{2}, \alpha^{1} ;(1,3,2)\right)$, and $\sigma$ is in cycle notation. Let $R(\omega)$ denote the rook placement corresponding to $\omega$. Define the $k$ th hit set of $B$ to be

$$
H_{k, N}(B)=\left\{R(\omega) \mid \omega \in C_{m} \prec S_{N} \text { and } \#(R(\omega) \cap B)=k\right\} .
$$

Also define the kth hit number of $B$ to be

$$
h_{k, N}=\# H_{k, N} .
$$

In order to show that two $m$-level rook equivalent Ferrers boards have the same hit numbers, we use Garsia and Milne's result again. To do so, we must construct a signed set and a sign reversing involution which has a set counted by $h_{k, N}$ as its fixed set. We do this as follows.

Let $N$ be large enough that $B$ fits inside $\mathrm{Sq}_{N, m}$, fix non-negative integers $i$ and $k$ with $i \leq k \leq N$. Then the set $S$ will consist of all configurations $C$ constructed as follows. Place $k$ black, $m$-level rooks $R$ on the board $B$. Furthermore, circle $i$ of the rooks. Finally, consider the the $N-k$ columns and $N-k$ levels which do not contain a black rook as a subboard of shape $\mathrm{Sq}_{N-k, m}$ and place $N-k$ white $m$-level rooks, denoted by $W$, on this subboard. Notice that, ignoring the color of the rooks, this is an $m$-level rook placement of $N$ rooks on $\mathrm{Sq}_{N, m}$. Thus it corresponds to some element of $C_{m} \backslash S_{N}$. Let the sign of a configuration be $(-1)^{i}$. See Figure 11 for two examples of such configurations. Here $m=2$ and $B=(1,2,4)$ is placed fitting in $\mathrm{Sq}_{3,2}$. On the left, there are no circled black rooks so $i=0$ and the white rooks are placed on the shaded subboard of shape $\mathrm{Sq}_{2,2}$. On the right there is one circled black rook so $i=1$ and the white rooks are on a shaded $\mathrm{Sq}_{1,2}$.

In order to produce a sign-reversing involution $I$ on such configurations $C$, we do the following. If $B$ contains neither a white rook nor a circled black rook, then $C$ is fixed by $I$. Otherwise, examine the columns of $B$ from left to right until the first white rook or circled black rook is found. If the first rook found is white, exchange it for a circled black rook and increase $i$ by 1 . If the first rook found is a circled black rook, exchange it for a white rook and decrease $i$ by 1 . See Figure 11 again for an illustration. It is easy to see that $I$ is an involution and reverses signs in its 2-cycles. Also note that fixed points have no circled black rooks, so $i=0$ and the sign of the configuration is +1 . Furthermore, there are no white rooks placed on $B$, so the $m$-level placement intersects $B$ in exactly $k$ black rooks. Thus the fixed points are exactly the elements of $H_{k, N}(B)$ if one just ignores the colors of the rooks. 

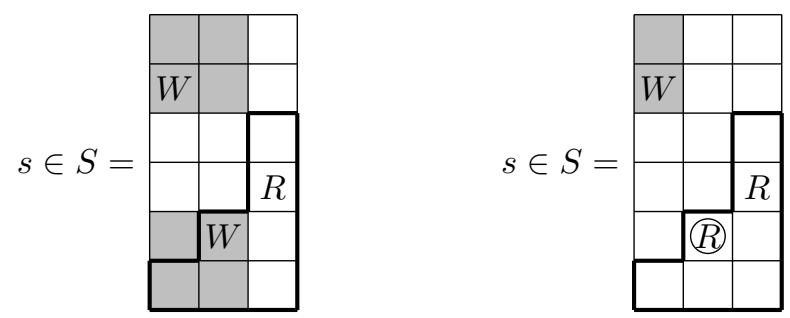

Fig. 11: On the left, an element in $S$ with sign +1 . On the right, the image under $I$ which has sign -1 .

The reader will find an example illustrating the next proof in Figure 12. This example uses the boards from the example of Theorem 12 found in Figure 6

Theorem 17 Let $B$ and $B^{\prime}$ be two m-level rook equivalent Ferrers boards and $N$ be large enough that $B$ and $B^{\prime}$ both fit inside $\mathrm{Sq}_{N, m}$. Then for any non-negative integer $k \leq N$, there is an explicit bijection between $H_{k, N}(B)$ and $H_{k, N}\left(B^{\prime}\right)$.

Proof: As in the proof of Theorem 15 we use the Garsia-Milne Involution Principle. Construct $S$ for $B$ placed inside $\mathrm{Sq}_{N, m}$ and $S^{\prime}$ for $B^{\prime}$ placed inside $\widehat{\mathrm{Sq}}_{N, m}^{\prime}$. Let $T$ be the fixed set of $I$ and $T^{\prime}$ be the fixed set for $S^{\prime}$.

To create a sign-preserving bijection $f$ from $S$ to $S^{\prime}$, consider an element $C \in S$. The black rooks, circled and uncircled, form an $m$-level rook placement of $k$ rooks on $B$. Map this to an $m$-level rook placement of $k$ rooks on $B^{\prime}$ using the explicit bijection guaranteed by Theorem 12 . Furthermore, add circles to the rooks on $B^{\prime}$ in such a way so that if the $r$ th rook from the right on board $B$ is circled, the $r$ th rook from the right on board $B^{\prime}$ is circled. Finally, place the white rooks on $\mathrm{Sq}_{N, m}^{\prime}$ by considering the subboard $\mathrm{Sq}_{N-k, m}^{\prime}$ of columns and levels containing no black rooks. Place the white rooks on this subboard in the exact same arrangement as they are in on the subboard $\mathrm{Sq}_{N-k, m}$ of $\mathrm{Sq}_{N, m}$.

Clearly the rooks, ignoring color, form an $m$-rook placement of $N$ rooks on $\mathrm{Sq}_{N, m}^{\prime}$ with $k$ black rooks on $B^{\prime}$, of which $i$ are circled. So this is an element of $S^{\prime}$. Additionally, since $i$ remains unchanged the map $f$ is sign preserving. Therefore the Involution Principle allows us to construct and explicit bijection between $T$ and $T^{\prime}$. Since there are bijections between $T$ and $H_{k, N}(B)$ and $T^{\prime}$ and $H_{k, N}\left(B^{\prime}\right)$, the compositions yields a bijection between that $H_{k, N}(B)$ and $H_{k, N}\left(B^{\prime}\right)$.

Corollary 18 Let $B$ and $B^{\prime}$ be two m-level rook equivalent Ferrers boards and $N$ be large enough such that $B$ and $B^{\prime}$ both fit inside $\mathrm{Sq}_{N, m}$. Then for any non-negative integer $k \leq N, h_{k, N}(B)=h_{k, N}\left(B^{\prime}\right)$.

Proof: Since $h_{k, N}(B)=\# H_{k, N}(B)$ and $h_{k, N}\left(B^{\prime}\right)=\# H_{k, N}\left(B^{\prime}\right)$, the bijection from Theorem 17 implies that $\# T=\# T^{\prime}$ and thus $h_{k, N}(B)=h_{k, N}\left(B^{\prime}\right)$.
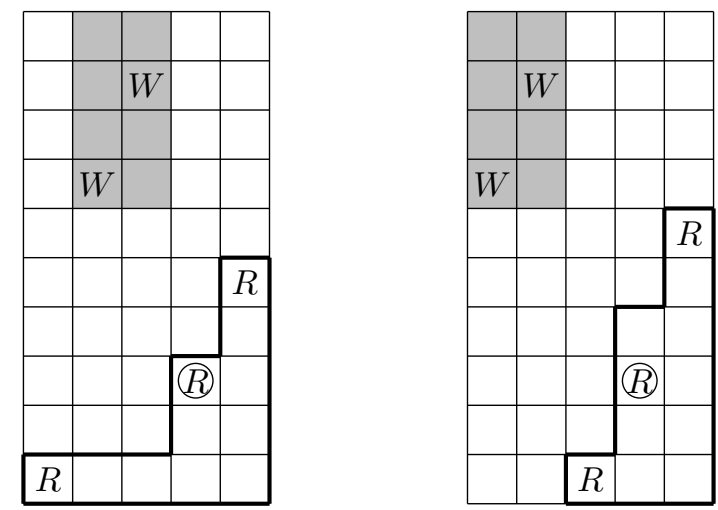

Fig. 12: On the left, a 2 -level placement of white rooks, black rooks, and circled black rooks on $(1,1,1,3,5)$ fit inside $\mathrm{Sq}_{5,2}$. On the right, the corresponding placement under the construction in Theorem 17 


\section{Open Problem}

The characterization of the rook equivalence class of a singleton board in terms of its root vector in Theorem 14 provides a way to count the number of singleton boards in a given $m$-level rook equivalence class. However, it is an open problem to count the number of Ferrers boards in a given $m$-level rook equivalence class. If $B_{S}$ is a singleton board, then perhaps counting the number of Ferrers boards $B$ with $l(B)=B_{S}$ would be a good start to this problem, but this too remains open.

\section{References}

[ABR01] Ron M. Adinh, Francesco Brenti, and Yuval Roichman. Descent numbers and major indices for the hyperoctahedral group. Adv. in Appl. Math., 27(2-3):210-224, 2001. Special issue in honor of Dominique Foata's 65th birthday (Philadelphia, PA, 2000).

[BLRS14] Kenneth Barrese, Nicholas Loehr, Jeffrey Remmel, and Bruce E. Sagan. $m$-Level rook placements. $J$. Combin. Theory Ser. A, 124:130-165, 2014.

[BR06] Karen S. Briggs and Jeffrey B. Remmel. $m$-rook numbers and a generalization of a formula of Frobenius to $C_{m} \nmid \mathcal{S}_{n}$. J. Combin. Theory Ser. A, 113(6):1138-1171, 2006.

[FS70] D. Foata and M. P. Schützenberger. On the rook polynomials of Ferrers relations. In Combinatorial theory and its applications, II (Proc. Colloq., Balatonfüred, 1969), pages 413-436. North-Holland, Amsterdam, 1970.

[GJW75] Jay R. Goldman, J. T. Joichi, and Dennis E. White. Rook theory. I. Rook equivalence of Ferrers boards. Proc. Amer. Math. Soc., 52:485-492, 1975.

[GM81] A. M. Garsia and S. C. Milne. Method for constructing bijections for classical partition identities. Proc. Nat. Acad. Sci. U.S.A., 78(4, part 1):2026-2028, 1981.

[KR46] Irving Kaplansky and John Riordan. The problem of the rooks and its applications. Duke Math. J., 13:259$268,1946$.

[LR09] Nicholas A. Loehr and Jeffrey B. Remmel. Rook-by-rook rook theory: bijective proofs of rook and hit equivalences. Adv. in Appl. Math., 42(4):483-503, 2009. 